\title{
Design Depth and Balanced Control System of an Autonomous Underwater Vehicle with Fuzzy logic
} Ahmad ZARKASI ${ }^{1}$, Endang Darmawan YUDI ${ }^{2}$, Muhammad Al RAVI ${ }^{3}$, Ilham Junias ANGKOTASAN $^{4}$

\author{
Izarkasi98@gmail.com, Intelligence System Research Group, Faculty of Computer Science, Sriwijaya University, \\ Palembang, Indonesia \\ ${ }^{2}$ endangdarmawanyudi123@gmail.com, Department of Computer Engineering, Faculty of Computer Science, \\ Sriwijaya University, Palembang, Indonesia \\ ${ }^{3}$ muhammad.al.ravi@gmail.com, Department of Computer Engineering, Faculty of Computer Science, Sriwijaya \\ University, Palembang, Indonesia \\ ${ }^{4}$ Ilhamjunius712@gmail.com, Department of Computer Engineering, Faculty of Computer Science, Sriwijaya \\ University, Palembang, Indonesia
}

\begin{abstract}
Underwater vehicles are the vehicle that can be moved and controlled in water by remotely. Autonomous Underwater Vehicle (AUV) is one type of underwater robot that is being developed lately. Unlike Remotely Operated Vehicle (ROV) which can be controlled manually using the remote. AUV can move automatically without being controlled directly by the user as long as the conditions of the robot program are met. With the movement and disturbance that is difficult to predict can make the stability of the robot one is very vulnerable, so it takes a condition that can stabilize the movement both at any depth. In practice, the on / off control system is still not able to balance the AUV properly if the robot is disturbed by water movement. Therefore, the purpose of this paper, we want to design a system using fuzzy logic with Takagi Sugeno method to stabilize its movement in the roll position and adjust the height of the robot from the bottom of the water. This study uses the IMU MPU6050 sensor hardware and sonar sensor as input, Arduino as a microcontroller and 2 brushed DC motors as actuators.
\end{abstract}

Keywords: Autonomous Underwater Robot, fuzzy logic controller, PWM

\section{INTRODUCTION}

Autonomous Underwater Vehicle (AUV) is an underwater diving robot that moves automatically without control, where the structure is very influential on the weight and balance of buoyancy and the force of gravity in the water. So we need a design that allows the robot to hold water and pressure in the water [1].

With the unpredictable movement of water and disturbances such as waves and water pressure can make the stability of the position of the robot one is very vulnerable, so it takes a condition that can make its movement remain stable both at any depth.

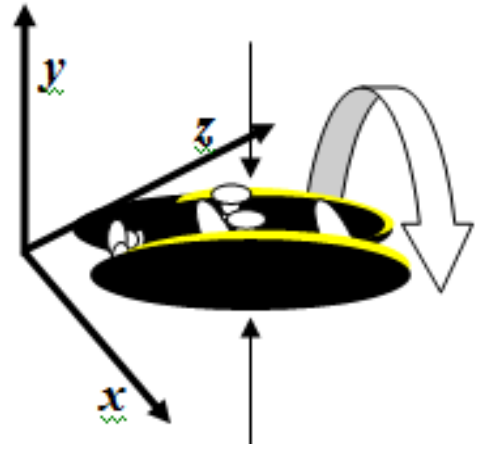

Figure 1. Body-fixed coordinate system
In practice, the on / off control system is still not able to balance the AUV properly if the robot is disturbed by water movement. Many ways that can be used to stabilize the position and adjust its depth, but in this study, we use fuzzy methods because the use is more flexible and easier to implement into the robot [2]. In this paper we using fuzzy logic with Takagi Sugeno method to stabilize its movement in the roll position and adjust the height of the robot from the bottom of the water. This study uses the IMU MPU6050 sensor hardware and sonar sensor as input, Arduino as a microcontroller and 2 brushed DC motors as actuators, with the input in the form of an error angle from the balance sensor reading both in the angle of the Accelerometer and Gyroscope and reading from the sensor sonar in the form of the value of the distance from the bottom of the water to the AUV will then be processed by a fuzzy logic control system that produces an output in the form of a motor rotation speed to return to a predetermined set point position.

\section{MODELING AND CONTROL AUV}

Autonomous Underwater Robot. In the AUV robot prototype, we will design using 2 motors to adjust 
the depth and adjust the balance in a roll [3]. Where the two motors will move opposite that the robot can move up and down in the water and to adjust the balance in the roll position. In some situations, these motors will move statically and dynamically, too much speed difference on motors will make the position of the robot not balanced.

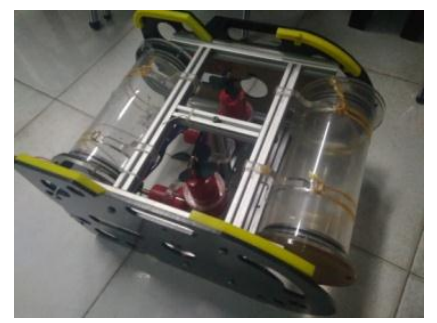

Figure 2. Prototype AUV

Designing AUV several things must be considered, among others: must have a little buoyancy so that when moving in the water the robot will not be pushed up. To reduce the use of motor energy (thrust upward) the force of attraction of the AUV must low [4].

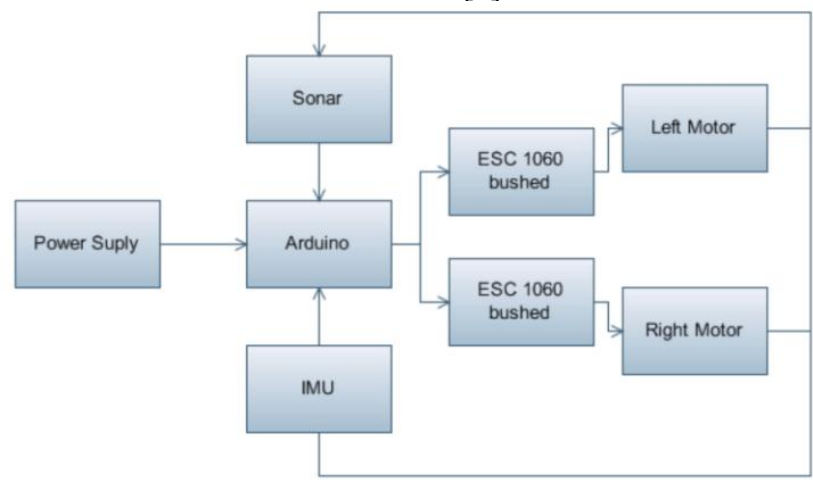

Figure 3. Desain schematic AUV

Sensor detection. The output of the accelerometer and gyroscope will produce the angles for the $\mathrm{x}, \mathrm{y}$ and $\mathrm{z}$ axes where. The $\mathrm{x}$-axis indicates a change in roll angle, $y$ indicates a change in pitch angle and $z$ indicates a change in the angle of yaw. Based on previous research [5] [6] uses the y-axis as a point to adjust the balance and adjust its depth, unfortunately, the resulting angle error is still large enough. For this reason, this paper will focus on the $\mathrm{X}$-axis (roll) as the center of the balance of motion of the robot. The output of the IMU MPU6050 sensor in the form of an angle between -90 to up to +90 on the $\mathrm{x}$-axis will be used as input for the Arduino to be processed so that it produces an output in the form of a PWM value. The negative value for the left axis and Positif value for the right axis.

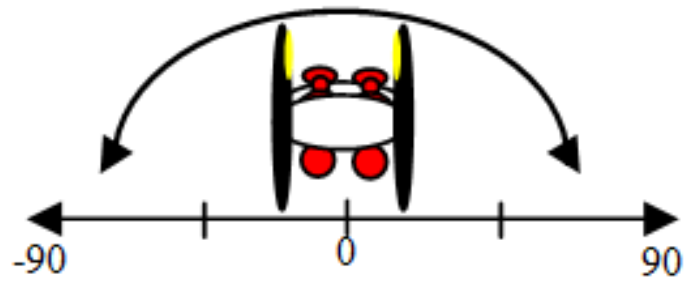

Figure 4. roll coordinate system

For sonar sensors, the value of the voltage coming out of the analog pin will be changed first to the ADC value so that it can be processed into a distance value by using the following equation;

$$
A D C=\frac{\text { Vin }}{\text { Vref }} 2^{n} \mathrm{ADC}=\frac{\text { Vin }}{\text { Vref }} 2^{\mathrm{n}}
$$

Where Vin represents the output voltage of the sensor (analog pin) and Vref represents the sensor input voltage (Vcc) and $n$ represents the magnitude of the data type.

Framework. The framework for Arduino in this research can be seen in Fig. 5. Software Arduino will process input data that has been obtained by using the fuzzy logic method. Read angle error by the IMU MPU6050 sensor and data from Sonar sensors will be sent to the controller. Then Arduino will send a value in the form of PWM in the motor driver to regulate the brushless motor speed with a propeller so that the desired slope angle and depth are following the setpoint that has been determined using the rules of fuzzy controller rules starting from fuzzification, rule-based, and defuzzification.

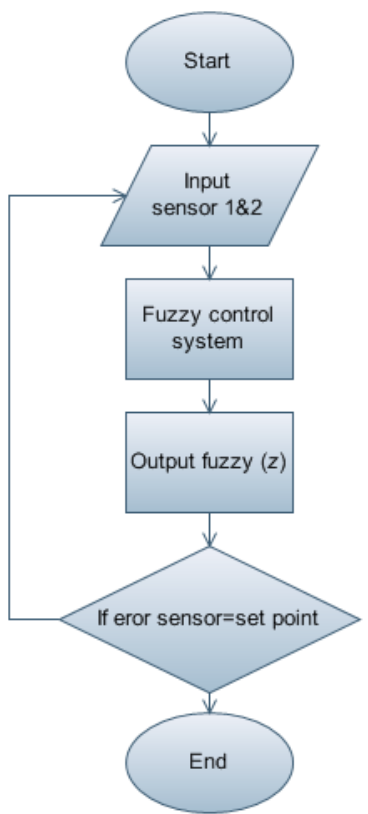

Figure 5. Desain schematic Arduino

If the slope angle and depth of AUV from the bottom of the water corresponds to the value of the setpoint, then the motor speed will remain and maintain the slope and altitude, but if it is not appropriate, it will read as an error and delta error which will be a reference of the Fuzzy controller thus changing the brushless motor speed back with propeller. The process will be repeated until the slope angle and altitude of the AUV are following the predetermined set point.

\section{MODELING CONTROL}

Fuzzy logic. Input crisp will be determined first in which category the membership function will then be fuzzification with the output in the form of $\mu_{\mathrm{x}}$ values for the degree of membership function input. The output fuzzification will be inferencing based on the rule-base. Finally, the data will be processed with the defuzzification equation with the output value in the form 
of PWM. where $x_{i}$ is the value of membership function from output and $\mathrm{Z}$ is the value of defuzzification result.

$\mathrm{Z}=\frac{\sum_{i=1}^{n}=\mu_{x i} \cdot x i}{\sum_{i=1}^{n}=\mu_{x i}}$

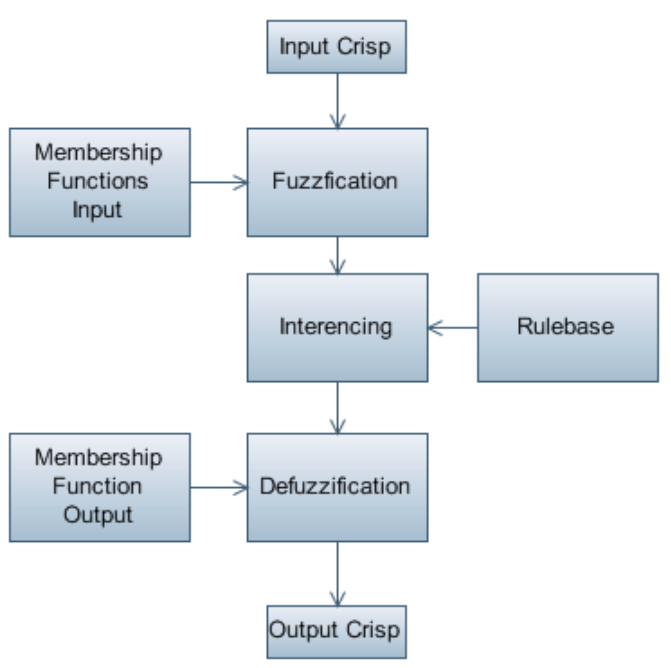

In this paper, we use the Sugeno method where output is in the form of a singleton set consisting of 5 sets. The control system is designed using 2 inputs, namely input from sonar (depth/distance) and input from IMU (slope). Where the input value received is the value of distance and angle position of each sensor, and the output (output) is a PWM value to set the two motors. Membership functions for input used are trapezoidal and triangular curves where fuzzy sets for input from sonar sensors (distance) are divided into 5 sets and the fuzzy set of IMU sensors (slope) is divided into 5 sets too.

Figure 6.Structure of fuzzy logic

Table 1. The initial and value of membership function

\begin{tabular}{|c|c|c|}
\hline Name & Initial & Range Value \\
\hline Very Fast Down & VFD & 1000 \\
\hline Fast Down & FD & 1200 \\
\hline Medium & $\mathrm{M}$ & 1600 \\
\hline Fast Up & FU & 1800 \\
\hline Very Fast Up & VFU & 2000 \\
\hline
\end{tabular}

\begin{tabular}{|c|c|c|}
\hline Name & Initial & Range Value $(\mathrm{cm})$ \\
\hline Very Near & VN & $0-40$ \\
\hline Near & $\mathrm{N}$ & $20-80$ \\
\hline Medium & $\mathrm{M}$ & $70-90$ \\
\hline Far & $\mathrm{F}$ & $80-140$ \\
\hline Very Far & VF & $>120$ \\
\hline
\end{tabular}

\begin{tabular}{|c|c|c|}
\hline Name & Initial & Range Value $\left(^{\circ}\right)$ \\
\hline Negative Large & NL & $-45-(-10)$ \\
\hline Negative Small & NS & $-20-0$ \\
\hline
\end{tabular}




\begin{tabular}{|c|c|c|}
\hline Zero & Z & $-5-5$ \\
\hline Positif Small & PS & $0-20$ \\
\hline Positif Large & PL & $10-45$ \\
\hline
\end{tabular}
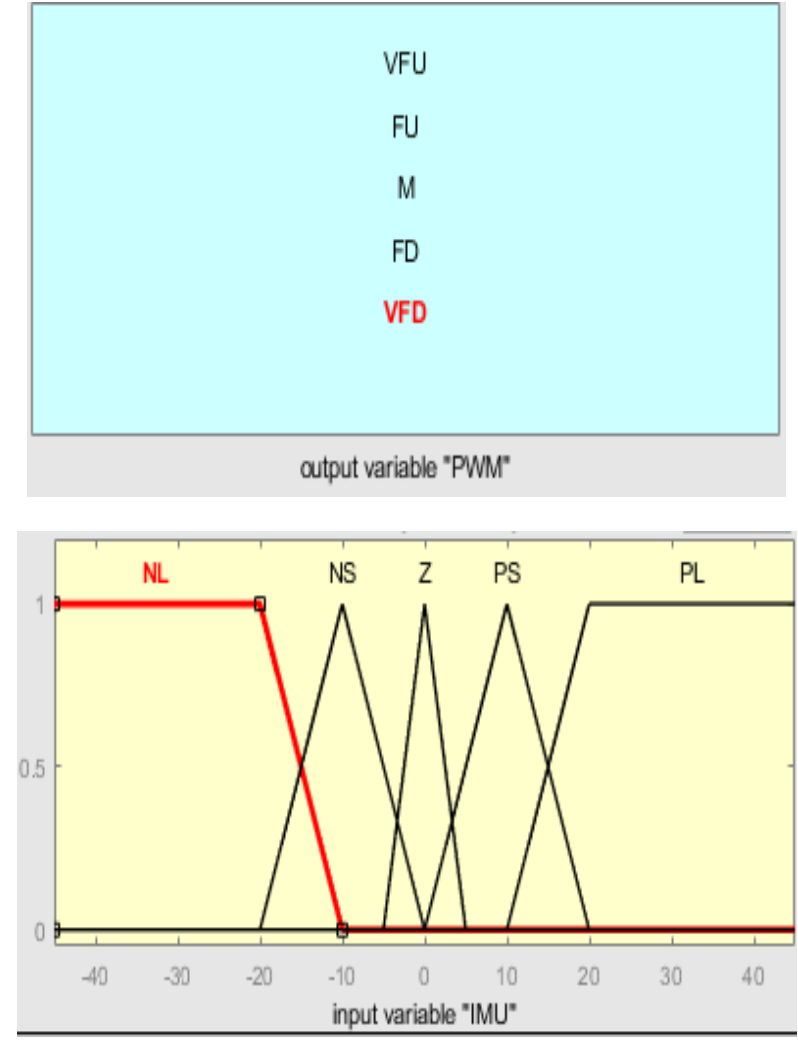

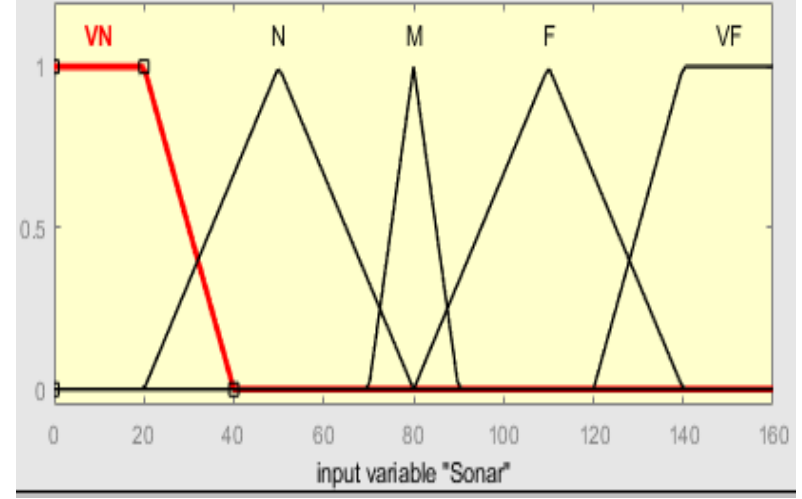

Figure 7. The membership function

To determine the number of rule-bases used is to multiply the number of membership sets so that the 25 rules can be obtained. In this paper we use the MIN (AND) implication method which then results from each rule can be seen in table 2 .

Table 2. Rule base.

\begin{tabular}{|c|c|c|c|c|c|}
\hline Sonar $/$ Imu & NL & NS & Z & PS & PL \\
\hline VN & VFD & FD & VFU & FD & VFD \\
\hline $\mathrm{N}$ & FD & FD & FU & FD & FD \\
\hline $\mathrm{M}$ & FD & FD & M & FD & FD \\
\hline F & FD & FD & FD & FD & FD \\
\hline VF & VFD & FD & VFD & FD & VFD \\
\hline
\end{tabular}

\section{MODELING AND CONTROL AUV}

Sensor IMU MPU6050. Connect the pins on the sensor to the Arduino pins based on the existing database. The MPU6050 sensor has a range of values for the accelerometer $\pm 2 \pm 4 \pm 8 \pm 16$ (datasheet). Sensor testing is compared with a protractor with a distance value from its angle, from $0 \mathrm{o}$ to $180 \mathrm{o}$. With the testing angle $-45^{\circ},-20^{\circ}$, $10^{\circ}, 0^{\circ}, 10^{\circ}, 20^{\circ}$, and $45^{\circ}$. Where the negative value to left sloping and the positive value to right sloping and the average error value obtained is $0,39 \%$. 
Table 3. Test result sensor IMU MPU6050.

\begin{tabular}{|c|c|c|c|}
\hline No & $\begin{array}{c}\text { Sensor IMU } \\
\left({ }^{\circ}\right)\end{array}$ & Protractor $\left(^{\circ}\right)$ & Error(\%) \\
\hline 1 & -45.62 & -45 & 0.62 \\
\hline 2 & -20.54 & -20 & 0.54 \\
\hline 3 & -10.29 & -10 & 0.29 \\
\hline 4 & 0.08 & 0 & 0.08 \\
\hline 5 & 10.14 & 10 & 0.14 \\
\hline 6 & 20.43 & 20 & 0.43 \\
\hline 7 & 45.68 & 45 & 0.68 \\
\hline \multicolumn{3}{|c|}{ Average } & 0.39 \\
\hline
\end{tabular}

Sonar Sensor. In this research, we use the ADC data value where the value is obtained from the equation above [4]. Because in this design we use Arduino, the max value of memory data (n) is 1023 (10 bits), with distance testing sensors 40,80, 120 and $160 \mathrm{~cm}$ where the average error value obtained is $2,25 \%$.

Table 4. Test result sensor Sonar.

\begin{tabular}{|l|ll|l|l|}
\hline No & $\begin{array}{l}\text { Sensor } \\
(\mathrm{cm})\end{array}$ & $\begin{array}{l}\text { Eonar } \\
(\mathrm{cm})\end{array}$ & Error(\%) \\
\hline 1 & 42 & 40 & 2 \\
\hline 2 & 79 & 80 & 1 \\
\hline 3 & 122 & 120 & 2 \\
\hline 4 & 164 & 160 & 4 \\
\hline \multicolumn{2}{|l|}{ Average } & 2.25 \\
\hline
\end{tabular}

Experiment to check the max and min error sonar sensor with a distance of $70 \mathrm{~cm}$ on fig 8 . Where the blue color is the distance of the sensor, the red

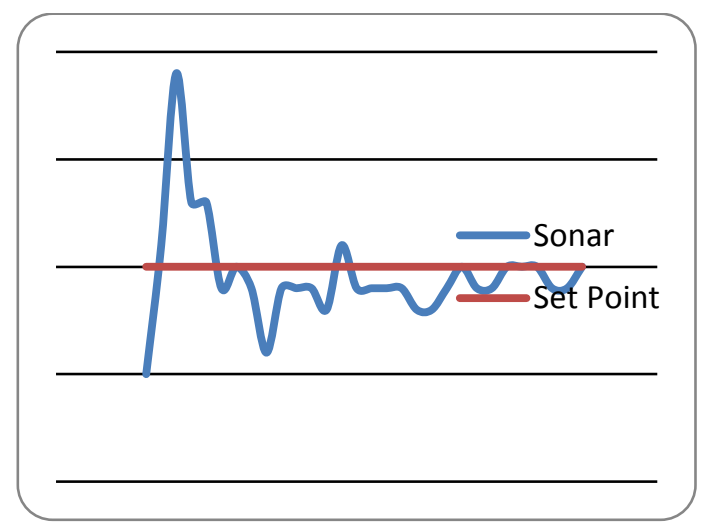

color is the setpoint of distance. The biggest error data is 89 and the minimum error data is 64 .

ESC 1060 Brushed. Testing is done by entering values from 1000 to 2000 , this value is obtained from previous research [7], the value of 1000 represents the reverse motor speed of 255 and 1500 represents 0 . Whereas from the range 1500 to 2000 represents the forward PWM value where 1500 represents 0 and 2000 represents 255 . If this equation is entered into excel then get the equation for PWM forward and backward as follows;

Figure 8. Results of testing sonar sensors. 


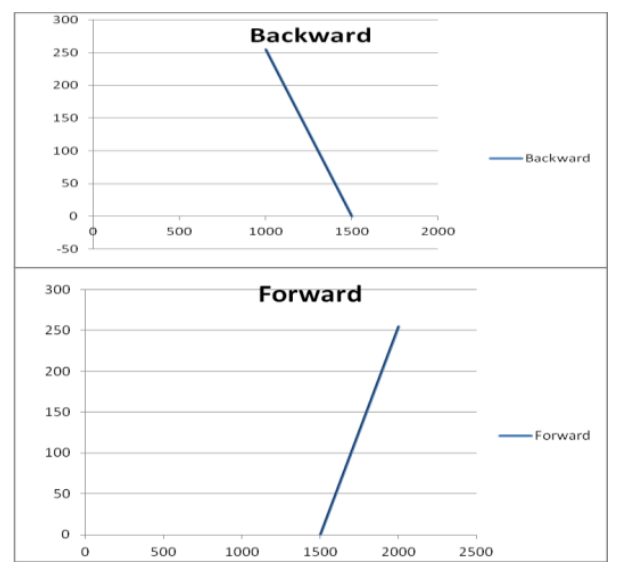

Figure 9. Result of backward and forwarding motor value
$\mathrm{Yb}=-0.51 \mathrm{X}+765$

(3)

Yf $=0.51 X-765$

where the value of $\mathrm{X}$ is the amount of data value from Arduino and $\mathrm{Y}$ is the value of the PWM.

Result Fuzzy. Testing fuzzy results are done by comparing fuzzy output values on Arduino with fuzzy values in Matlab. Because for AUV it is still in the design stage, then inputting the values on Arduino and Matlab manually. Where the value tested and the results can be seen in Table 5. Where negative value is left sloping of AUV and a positive value is the right sloping of AUV.

Table 5. result from Output fuzzy from Arduino and Matlab

\begin{tabular}{|c|c|c|l|l|l|}
\hline $\mathrm{No}$ & $\begin{array}{c}\text { Sonar } \\
(\mathrm{cm})\end{array}$ & Imu $\left(^{\circ}\right)$ & Arduino & Matlab & $\begin{array}{c}\text { Error } \\
(\%)\end{array}$ \\
\hline 1 & 25 & -18 & 1083.12 & 1070 & 1,21 \\
\hline 2 & 30 & -12 & 1167.57 & 1180 & 1,05 \\
\hline 3 & 36 & 15 & 1171.43 & 1170 & 0,12 \\
\hline 4 & 46 & 1 & 1733.33 & 1730 & 0,19 \\
\hline 5 & 74 & -4 & 1400.00 & 1360 & 2,86 \\
\hline 6 & 78 & 8 & 1200.00 & 1200 & 0 \\
\hline 7 & 83 & 2 & 1460 & 1440 & 1,36 \\
\hline 8 & 85 & -4 & 1285.71 & 1300 & 1,1 \\
\hline 9 & 125 & 23 & 1133.33 & 1130 & 0,29 \\
\hline 10 & 130 & -4 & 1164.71 & 1160 & 0,4 \\
\hline Average & & & & 0,858 \\
\hline
\end{tabular}

From these results, it can be seen that the error value is not too much different, with the biggest error is $2,86 \%$.

\section{CONCLUSION}

From the tests that have been done, both from the sensor test and the fuzzy value test found that the error value is not too large, for the IMU average error sensor obtained is $0.39 \%$ and for Sonar sensor is $2.25 \%$. In testing fuzzy results, the differences between fuzzy Arduino results and the results of fuzzy Matlab are not very different. With the biggest error is $2,86 \%$ and to average error the sensor is $0,85 \%$.

Figure 10. Test result Output fuzzy from Arduino and Matlab 


\section{ACKNOWLEDGMENT}

The experiment to validate functions controller that has been designed gets a satisfactory result. By analyzing our results conclude that is possible to control the AUV with fuzzy controllers. But now it's impossible to compare our results with other existing control approaches because experiments are done inside very limited settings. There is no guarantee that the controller is designed optimally. We strongly believe that further improvements will be needed after successfully implemented in the form of hardware.

\section{REFERENCES}

[1] S. G. Anavatti, P. P. Wong, and J. Y. Choi, "Design and implementation of fuzzy logic controller for wing rock," Int. J. Control. Autom. Syst., vol. 2, no. 4, pp. 494-500, 2004.

[2] S. K. Jain, S. Bora, and M. Singh, "A Review Paper on: Autonomous Underwater Vehicle," Int. J. Control. Autom. Syst., vol. 6, no. 2, pp. 38-40, 2015.

[3] S. Yadav and P. S. S. Patil, "Design and Implementation of Fuzzy Logic Controllers for DC Motor Speed Control using MATLAB-GUI Application .," Int. J. Control Autom.., vol. 3, no. 2, pp. 725-728, 2013.

[4] D. D. Debrianto, Suwandi, R. F. Iskandar, "Static StabilityControl Design in Autonomous Underwater Vehicle Using Fuzzy Logic Method ," Universitas Telkom, 2018.

[5] A. Nag, S. S. Patel, and S. A. Akbar, "Fuzzy logic based depth control of an autonomous underwater vehicle," Proc. - 2013 IEEE Int. Multi Conf. Autom. Comput. Control. Commun. Compress. Sensing, iMac4s 2013, pp. 117-123, 2013.

[6] L. K. Wardhani and E. Haerani, "Analisis Pengaruh Pemilihan Fuzzy Membership Function Terhadap Output Sebuah Sistem Fuzzy Logic," Uin Suska., pp. 326-333, 2011.

[7] Y. Zhuoyi, S. Yumin, and S. Hailong, "Vehicle Made of Composite Material," Harbin, China., pp. 0-3, 2014. 